\title{
Propagation Properties of Vortex Cosine- Hyperbolic-Gaussian Beams Through Oceanic Turbulence
}

\section{Mohamed Lazrek}

Chouaib Doukkali University Faculty of Sciences: Universite Chouaib Doukkali Faculte des Sciences Zoubir Hricha

Chouaib Doukkali University Faculty of Sciences: Universite Chouaib Doukkali Faculte des Sciences Abdelmajid Belafhal ( $\square$ belafhal@gmail.com)

Chouaib Doukkali University https://orcid.org/0000-0003-2735-3108

\section{Research Article}

Keywords: Vortex cosine-hyperbolic-Gaussian beam, Oceanic turbulence, Laser Propagation, Average intensity.

Posted Date: November 9th, 2021

DOI: https://doi.org/10.21203/rs.3.rs-1054533/v1

License: (a) (1) This work is licensed under a Creative Commons Attribution 4.0 International License. Read Full License

Version of Record: A version of this preprint was published at Optical and Quantum Electronics on February 17th, 2022. See the published version at https://doi.org/10.1007/s11082-022-03541-x. 


\title{
Propagation Properties Of Vortex Cosine-Hyperbolic-Gaussian Beams Through Oceanic Turbulence
}

\author{
M. Lazrek, Z. Hricha*, A. Belafhal* \\ Laboratory LPNAMME, Laser Physics Group, \\ Department of Physics, Faculty of Sciences, Chouaïb Doukkali University, \\ P. B 20, 24000 El Jadida, Morocco \\ *Corresponding authors.E-mail: belafhal@gmail.com, hrichazo66@gmail.com
}

\begin{abstract}
Based on the extended Huygens-Fresnel diffraction integral, the analytical expression of the average intensity for a vortex cosine hyperbolic-Gaussian beam ( $\mathrm{vChGB}$ ) propagating in oceanic turbulence is derived in detail. From the derived formula, the propagation properties of a vChGB in oceanic turbulence, including the average intensity distribution and the beam spreading are discussed with numerical examples. It is shown that oceanic turbulence influences strongly the propagation properties of the beam in the turbulent medium. The vChGB may propagate within shorter distance in weak oceanic turbulence by increasing the dissipation rate of mean-square temperature and the ratio of temperature to salinity fluctuation or by increasing the dissipation rate of turbulent kinetic energy per unit mass of sea water. Meanwhile, the evolution properties of the vChGB in the oceanic turbulence are affected by the initial beam parameters, namely the decentered parameter $b$, the topological charge $M$, the beam waist width $\omega_{0}$ and the wavelength $\lambda$. The obtained results can be beneficial for applications in optical underwater communication and remote sensing domain, imaging, and so on.
\end{abstract}

Keywords: Vortex cosine-hyperbolic-Gaussian beam; Oceanic turbulence; Laser Propagation; Average intensity.

\section{Introduction}

In last few years, the propagation behavior of laser beams in oceanic turbulence has attracted much attention due to their potential applications in underwater wireless optical communication and imaging systems [1-5]. The influence of temperature and salinity fluctuations on propagation of laser beams, including the degree of polarization, mutual coherence function, spreading, and the scintillation index, have widely been investigated. Up to now, the propagation properties of various types of laser beams in an oceanic environment have been reported, such as those for radially 
polarized Gaussian beams [6], Gaussian Schell-model vortex beam [7], stochastic electromagnetic vortex beams [8], partially coherent flat-topped vortex hollow beam [9], partially coherent annular decentered beams [10], partially coherent Hermite-Gaussian linear array beams [11], hollow Gaussian beams [12], cosine-Gaussian-correlated Schell-model beams [13], partially coherent Lorentz-Gauss vortex beams [14], partially coherent four-petal Gaussian vortex beams [15], random electromagnetic multi-Gaussian Schell-model vortex beam [16], flat-topped beams [17] and Airy beams with power exponential phase vortex [18]. Besides, a new beam model named vortex-cosh-Gaussian beam (vChGB) has been freshly investigated by us [19]. The beam possesses three key parameters, namely the beam order, the decentered parameter $b$ and the vortex charge number $M$. The beam parameters can be adjusted to control broadly the beam intensity distribution pattern, the beam size and the central dark spot in the initial plane. The beam profile is generally hollow dark array-like, but can also describe for special values of the beam parameters many known laser beams, such as the vortex-Gaussian beam [20], cosh-Gaussian beam [21], and fundamental Gaussian beam [22]. The propagation properties of vChGBs through different optical media have been investigated [19, 23-24]. However, to the best of our knowledge, the propagation of vHChGBs in the oceanic turbulence has not been reported, thus, this will be the subject of the present work. We aim in this paper to investigate the influence of oceanic turbulence on the propagation properties of a vChGB. The evolution properties of the beam through oceanic turbulence, and the effects of the turbulence strength of the sea-water in addition to the initial beam parameters on the average intensity distribution are investigated numerically and theoretically. In the second Section of the manuscript, a theoretical analysis based on the extended Huygens-Fresnel integral and Rytov method for the propagation of a $\mathrm{vChGB}$ in oceanic turbulence is made, and the corresponding analytical expression of the average intensity distribution is derived. In Section 3, the evolution behaviour of the intensity distribution and the spreading of a vChGB in oceanic turbulence are analysed with illustrative numerical examples. The main results obtained are highlighted in the conclusion part.

\section{Propagation of a vChGB through oceanic turbulence}

In the Cartesian coordinate system, a vChGB propagating along the z-axis in the source plane $\mathrm{z}=0$ can be written as [19] 
$E\left(r_{0}, z=0\right)=\cosh \left(b \frac{x_{0}}{\omega_{0}}\right) \cosh \left(b \frac{y_{0}}{\omega_{0}}\right) e^{-\left(\frac{x_{0}^{2}+y_{0}^{2}}{\omega_{0}^{2}}\right)}\left(x_{0}+i y_{0}\right)^{M}$,

where $r_{0}=\left(x_{0}, y_{0}\right)$ is the position vector at the source plane and $\omega_{0}$ is the waist radius of the Gaussian part. $b$ is the decentered parameter associated to the cosh part. $M$ is the topological charge of the vChGB.

Within the framework of the paraxial approximation, and according to the extended HuygensFresnel diffractionintegral, the propagation of a vChGB through the oceanic turbulence along the $\mathrm{z}$-axis can be formulated as [25-26]

$E(\rho, z)=-\frac{i k}{2 \pi z} \exp (-i k z) \int_{-\infty}^{+\infty} \int_{-\infty}^{+\infty} E\left(\rho_{0}, 0\right) \exp \left[-\frac{i k}{2 z}\left(\rho_{0}-\rho\right)^{2}+\psi\left(\rho_{0}, \rho, z\right)\right] d r_{0}$,

where $\tilde{r}=(x, y)$ are the transverse coordinates in the receiver plane, $z$ is the distance between the initial plane $\mathrm{z}=0$ and the receiver plane, and $d \breve{r}_{0}=d x_{0} d y_{0}$ is the elementary surface in the initial plane $z=0 . \psi\left(r_{0}, r, z\right)$ is the Rytov solution that represents the random part for the complex phase of a spherical wave spreading from the source plane to the output plane, $k=\frac{2 \pi}{\lambda}$ is the wave number and $\lambda$ is the wavelength.

The average intensity of $\mathrm{vChGB}$ propagating in the receiver plane can be written as $\langle I(\rho, z)\rangle=\langle E(r, z) E *(r, z)\rangle$,

where $*$ and $\langle$.$\rangle denote the complex conjugation and the ensemble average over the medium$ statistics, respectively.

Substituting Eq. (2) into Eq. (3), one can obtain

$$
\begin{aligned}
\langle I(\rho, z)\rangle & =\frac{k^{2}}{4 \pi^{2} z^{2}} \int_{-\infty}^{+\infty} \int_{-\infty}^{+\infty} \int_{-\infty}^{+\infty} \int_{-\infty}^{+\infty} E_{0}\left(x_{01}, y_{01}, 0\right) E_{0} *\left(x_{02}, y_{02}, 0\right) \\
& \times \exp \left[-\frac{i k}{2 z}\left\{\left(x_{01}-x\right)^{2}+\left(y_{01}-y\right)^{2}-\left(x_{02}-x\right)^{2}-\left(y_{02}-y\right)^{2}\right\}\right] \\
& \times\left\langle\exp \left[\psi\left(\rho_{01}, \rho\right)+\psi *\left(\rho_{02}, \rho\right)\right]\right\rangle d \rho_{01} d \rho_{02},
\end{aligned}
$$

$d r_{0 i}=d x_{0 i} d y_{0 i}$ (with $i=1$ or 2 ) are elementary surfaces in the initial plane $z=0$.

The last term in angle brackets of Eq. (4) can be expressed as [26]

$$
\left\langle\exp \left[\psi\left(x_{01}, y_{01}, x, y\right)+\psi *\left(x_{02}, y_{02}, x, y\right)\right]\right\rangle=\exp \left\{-\frac{1}{\rho_{0}^{2}}\left[\left(x_{01}-x_{02}\right)^{2}+\left(y_{01}-y_{02}\right)^{2}\right]\right\},
$$


where $\rho_{0}=\left[\frac{\pi^{2} k^{2} z}{3} \int_{0}^{+\infty} d \kappa \kappa^{3} \Phi(\kappa)\right]^{-1 / 2}$ is the coherence length of a spherical wave propagating in oceanic turbulence, with $\kappa$ is the spatial frequency, and $\Phi(\kappa)$ is the one-dimensional spatial power spectrum of the refractive index fluctuations, which for an homogeneous and isotropic turbulent ocean is given by [1]

$\Phi(\kappa)=0.388 \times 10^{-8} \varepsilon^{-11 / 3}\left[1+2.35(\kappa \eta)^{2 / 3}\right] f\left(\kappa, \varsigma, \chi_{T}\right)$,

where $\varepsilon$ is the rate of dissipation of turbulent kinetic energy per unit mass of fluid, which may vary in the range from $10^{-10} \mathrm{~m}^{2} \mathrm{~s}^{-3}$ to $10^{-1} \mathrm{~m}^{2} \mathrm{~s}^{-3} \cdot \eta=10^{-3}$ is the Kolmogorov inner scale, and

$$
f\left(\kappa, \varsigma, \chi_{T}\right)=\frac{\chi_{T}}{\varsigma^{2}}\left[\varsigma^{2} \exp \left(-A_{T} \delta\right)+\exp \left(-A_{S} \delta\right)-2 \varsigma \exp \left(-A_{T S} \delta\right)\right]
$$

with $\chi_{T}$ is the rate of dissipation of mean square temperature varying in the range from $10^{-4} \mathrm{~K}^{2} \mathrm{~s}^{-1}$ to $\quad 10^{-10} K^{2} s^{-1}, \quad \delta=8.284(\kappa \eta)^{4 / 3}+12.978(\kappa \eta)^{2}, A_{T}=1.863 \times 10^{-2}, \quad A_{S}=1.9 \times 10^{-4}, \quad$ and $A_{T S}=9.41 \times 10^{-3}, \varsigma$ describes the relative strength of temperature and salinity fluctuations, which in the ocean water varies in the range from -5 to 0 . The zero value corresponding to the case when salinity-driven turbulence dominates, and -5 value describes the case when temperature-driven turbulence prevails.

Substituting Eq. (1) into Eq. (4), and using the binomial formula [27]:

$$
\left(x_{0}+i y_{0}\right)^{m}=\sum_{l=0}^{m} C_{l}^{m} x_{0}^{l}\left(i y_{0}\right)^{m-l},
$$

where

$$
C_{l}^{m}=\frac{m !}{l !(m-l) !}
$$

and after rearanging the integrand function, one can obtain

$$
\langle I(\rho, z)\rangle=\left(\frac{k}{2 \pi z}\right)^{2} \sum_{l=0}^{M} C_{l}^{M} \sum_{n=0}^{M} C_{n}^{M} W_{l, n}(x, z) W_{M-l, M-n}(y, z),
$$

where

$$
\begin{gathered}
W_{l, n}(v, z)=\int_{-\infty}^{+\infty} \int_{-\infty}^{+\infty} v_{01}^{l} v_{02}^{n} \cosh \left(\frac{b v_{01}}{\omega_{0}}\right) \cosh \left(\frac{b v_{02}}{\omega_{0}}\right) \exp \left(-\alpha v_{01}^{2}+\frac{i k v}{z} v_{01}\right) \\
\quad \times \exp \left\{-\alpha^{*} v_{02}^{2}+\left(-\frac{i k v}{z}+\frac{2 v_{01}}{\rho_{0}^{2}}\right) v_{02}\right\} d v_{01} d v_{02}
\end{gathered}
$$


in which $v$ represents either $x$ or $y$, and $\alpha$ is the auxiliary parameter given by

$$
\alpha=\frac{i k}{2 z}+\frac{1}{\omega_{0}^{2}}+\frac{1}{\rho_{0}^{2}}
$$

Eq. (10) can be rewritten in the form

$W_{l, n}(v, z)=\int_{-\infty}^{+\infty} v_{01}^{l} \cosh \left(\frac{b v_{01}}{\omega_{0}}\right) \exp \left(-\alpha v_{01}^{2}+\frac{i k v}{z} v_{01}\right) Q_{n}\left(v_{01}, z\right) d v_{01}$,

where

$Q_{n}\left(v_{01}, z\right)$ is an one-variable integral expression given by

$Q_{n}\left(v_{01}, z\right)=\int_{-\infty}^{+\infty} v_{02}^{n} \cosh \left(\frac{b v_{02}}{\omega_{0}}\right) \exp \left\{-\alpha^{*} v_{02}^{2}+\left(-\frac{i k v}{z}+\frac{2 v_{01}}{\rho_{0}^{2}}\right) v_{02}\right\} d v_{02}$.

Using the explicit form of $\cosh$ function: $\cosh (u)=\frac{1}{2}[\exp (u)+\exp (-u)]$, Eq. (13) can be expressed as

$$
Q_{n}\left(v_{01}, z\right)=\frac{1}{2}\left[Q_{n}^{+}\left(v_{01}, z\right)+Q_{n}^{-}\left(v_{01}, z\right)\right]
$$

with

$Q_{n}^{ \pm}\left(v_{01}, z\right)=\int_{-\infty}^{+\infty} v_{02}^{n} \exp \left\{-\alpha^{*} v_{02}^{2}+2 \beta_{v 01} v_{02}\right\} d v_{02}$,

and

$$
\beta_{v 01}=-\frac{i k v}{2 z}+\frac{v_{01}}{\rho_{0}^{2}} \pm \frac{b}{2 \omega_{0}} .
$$

By using the following integral equation [28]

$$
\int_{-\infty}^{+\infty} x^{n} e^{-p x^{2}+2 q x} d x=e^{\frac{q^{2}}{p}} \sqrt{\frac{\pi}{p}}\left(\frac{1}{2 i \sqrt{p}}\right)^{n} H_{n}\left(\frac{i q}{\sqrt{p}}\right),
$$

where $H_{m}($.$) is the Hermite polynomial of m-order, the integral of Eq. (15a) is carried out, and$ Eq. (10) can be expressed as

$$
\begin{aligned}
& W_{l, n}(v, z)=\frac{1}{2} \sqrt{\frac{\pi}{\alpha^{*}}}\left(\frac{1}{2 i \sqrt{\alpha^{*}}}\right)^{n} \int_{-\infty}^{+\infty} v_{01}^{l} \cosh \left(\frac{b v_{01}}{\omega_{0}}\right) \exp \left(-\alpha v_{01}^{2}+\frac{i k v}{z} v_{01}\right) \\
& {\left[\exp \left\{\frac{\left(\beta_{v 01}^{+}\right)^{2}}{\alpha^{*}}\right\} H_{n}\left(\frac{i \beta_{v 01}^{+}}{\sqrt{\alpha^{*}}}\right)+\exp \left\{\frac{\left(\beta_{v 01}^{-}\right)^{2}}{\alpha^{*}}\right\} H_{n}\left(\frac{i \beta_{v 01}^{-}}{\sqrt{\alpha^{*}}}\right)\right] d v_{01} }
\end{aligned}
$$

Now, by recalling the following series expansions of the Hermite polynomial [29], 


$$
H_{m}(x)=\sum_{j=0}^{[m / 2]} \frac{(-1)^{j} m !}{j !(m-2 j) !}(2 x)^{m-2 j}
$$

and after performing tedious but straightforward integration, Eq. (17) yields

$$
\begin{aligned}
& W_{l, n}(v, z)=\frac{1}{4} \sqrt{\frac{\pi}{\alpha^{*}}} \sqrt{\frac{\pi}{a}}\left(\frac{1}{2 i \sqrt{\alpha^{*}}}\right)^{n} \sum_{s=0}^{\left[\frac{n}{2}\right]} \frac{(-1)^{s} n !}{s !(n-2 s) !}\left(\frac{2 i}{\sqrt{\alpha^{*}}}\right)^{n-2 s} \sum_{j=0}^{n-2 s} C_{j}^{n-2 s}\left(\frac{1}{2 i \sqrt{a}}\right)^{l+j}\left(\frac{1}{\rho_{0}^{2}}\right)^{j} \\
& \times\left\{\begin{array}{l}
\exp \left\{\frac{1}{\alpha^{*}}\left(\frac{-i k v}{2 z}+\frac{b}{2 \omega_{0}}\right)^{2}\right\}\left[\exp \left\{\frac{\left(f_{l v}^{+}\right)^{2}}{a}\right\} H_{l+j}\left(\frac{i f_{l v}^{+}}{\sqrt{a}}\right)+\exp \left\{\frac{\left(f_{l v}^{-}\right)^{2}}{a}\right\} H_{l+j}\left(\frac{i f_{l v}^{-}}{\sqrt{a}}\right)\right] \\
\left.+\exp \left\{\frac{1}{\alpha^{*}}\left(\frac{-i k v}{2 z}-\frac{b}{2 \omega_{0}}\right)^{2}\right\}\left[\exp \left\{\frac{\left(f_{2 v}^{+}\right)^{2}}{a}\right\} H_{l+j}\left(\frac{i f_{2 v}^{+}}{\sqrt{a}}\right)+\exp \left\{\frac{\left(f_{2 v}^{-}\right)^{2}}{a}\right\} H_{l+j}\left(\frac{i f_{2 v}^{-}}{\sqrt{a}}\right)\right]\right\},
\end{array}\right.
\end{aligned}
$$

with

$$
\begin{aligned}
& a=\alpha-\frac{1}{\rho_{0}{ }^{4} \alpha^{*}}, \\
& f_{1 v}^{ \pm}=\frac{1}{\rho_{0}^{2} \alpha^{*}}\left(\frac{-i k v}{2 z}+\frac{b}{2 \omega_{0}}\right)+\left(\frac{i k v}{2 z} \pm \frac{b}{2 \omega_{0}}\right),
\end{aligned}
$$

and

$f_{2 v}^{ \pm}=\frac{1}{\rho_{0}^{2} \alpha^{*}}\left(\frac{-i k v}{2 z}-\frac{b}{2 \omega_{0}}\right)+\left(\frac{i k v}{2 z} \pm \frac{b}{2 \omega_{0}}\right)$.

The direct substituting from Eq. (19) into Eq. (9) will give the analytical expression of a vChGB propagating in oceanic turbulence. The obtained formula depends on the oceanic turbulence strength and the initial beam parameters in addition to the propagation distance z. It will allow us to analyse the propagation properties of $\mathrm{vChGBs}$ propagating in oceanic turbulence.

From the main analytical formula derived above, one can distinguish the special cases following:

-The case $b=0$, Eq. (9) will give the corresponding formula for the hollow Gaussian vortex beam, which can be expressed as

$$
\langle I(\rho, z)\rangle=\left(\frac{k}{2 z}\right)^{2} \frac{1}{\alpha^{*} a}\left(\frac{1}{2 i \sqrt{\alpha^{*}}}\right)^{M} \sum_{l=0}^{M} C_{l}^{M} \sum_{n=0}^{M} C_{n}^{M} R_{l, n}(x, z) R_{M-l, M-n}(y, z),
$$

where 


$$
\begin{aligned}
& R_{l, n}(v, z)=\exp \left(B v^{2}\right)_{s=0}^{\left[\frac{n}{2}\right]} \frac{(-1)^{s} n !}{s !(n-2 s) !}\left(\frac{2 i}{\sqrt{\alpha^{*}}}\right)^{n-2 s} \sum_{j=0}^{n-2 s} C_{j}^{n-2 s}\left(\frac{1}{2 i \sqrt{a}}\right)^{l+j}\left(\frac{1}{\rho_{0}^{2}}\right)^{j} H_{l+j}\left(\frac{i A}{\sqrt{a}} v\right), \\
& A=\frac{i k}{2 z}\left(1-\frac{1}{\alpha^{*} \rho_{0}^{2}}\right),
\end{aligned}
$$

and

$$
B=\frac{A^{2}}{a}-\frac{k^{2}}{4 \alpha^{*} z^{2}}
$$

It should be noted that we have checked numerically that Eq. (21) is consistent with Eq. (16) of Ref. [12] even though they are different in the form.

-The case when $M=0$, Eq. (9) will yield the propagation equation for the cosh-Gaussian beam in oceanic turbulence.

\section{Numerical examples and analysis}

In this section, based on the formulas derived above, the evolution properties of a vChGB propagating through the oceanic turbulence are illustrated with numerical calculations. As is previously indicated, the initial vChGB can have two profile types depending on the value the parameter $b$ : the initial beam is Gaussian-like for small values of $b$ (typically when $\mathrm{b}=0.1$ ) while for a large value of $\mathrm{b}$ (typically, $b=4$ ), the profile is four-petal like. Consequently, in the following, the two initial beam configurations are examined, separately. Unless it is specified, the calculation parameters are set arbitrarily as $\omega_{0}=2 \mathrm{~cm}, M=1, \lambda=417 \mathrm{~nm}, \varepsilon=10^{-7}, \chi_{T}=10^{-9}$ and $\varsigma=-2.5$.

Figs. 1 and 2 show the normalized average intensity, depicted with both contour graphs and with one dimensional representation, of the initial $\mathrm{vChGBs}$ propagating through oceanic turbulence for the two initial beam configurations. From the plots in the top row of Fig. 1, one can see that a beam with small $b$ configuration can keep its dark center at short propagation distances (see plot (a.1)), but as the propagation distance is further increased, the beam will lose gradually its initial hollow dark-like profile and evolves into Gaussian-like beam (see plot a3-a5). The plots in bottom row of Fig. (1) show that the beam with large $b$ configuration can retain its four-petal profile for the first transmission process, but with increasing the propagation distance the light petals get closer and bond, and finally in the far-field the beam morphed into a flat-topped profile-like (see plots Fig. 1(b.5) and Fig. 2 (2b)). These results demonstrate that the propagation of a vChGB is influenced by the turbulent medium with respect to that corresponding to the free space. 


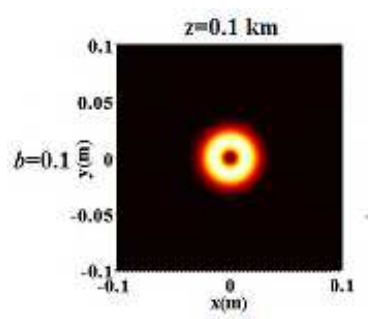

(a.1)

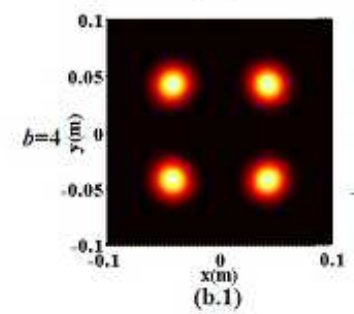

$t=0.3 \mathrm{~km}$
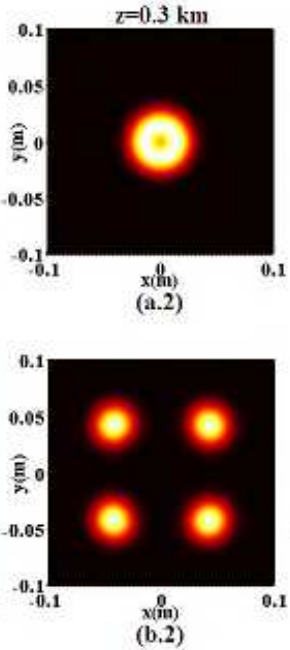

$2=0.6 \mathrm{~km}$
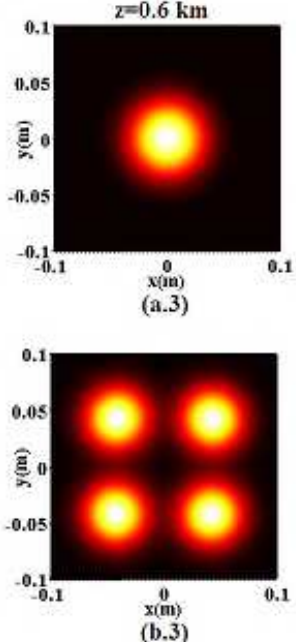

$z=0.9 \mathrm{~km}$

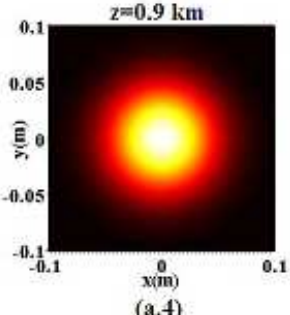

(a.4)

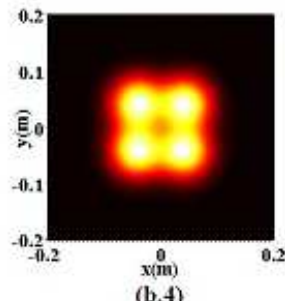

$z=1.2 \mathrm{~km}$
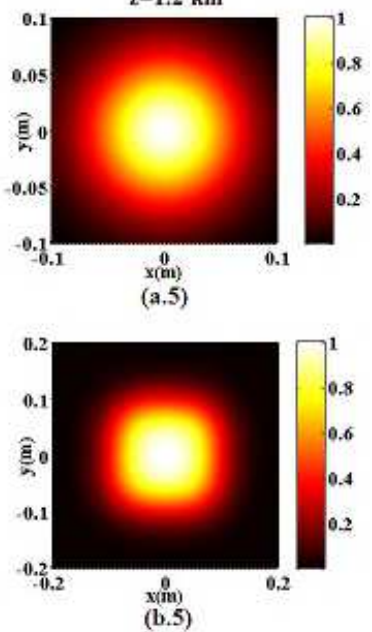

Figure 1: Normalized intensity of a $\mathrm{vChGB}$ with $M=1$ in oceanic turbulence for $z=0.1 \mathrm{~km}, 0.3 \mathrm{~km}, 0,6 \mathrm{~km}, 0.9 \mathrm{~km}$ and $1.2 \mathrm{~km}$. Top row for $b=0.1$, and bottom row for $b=4$.
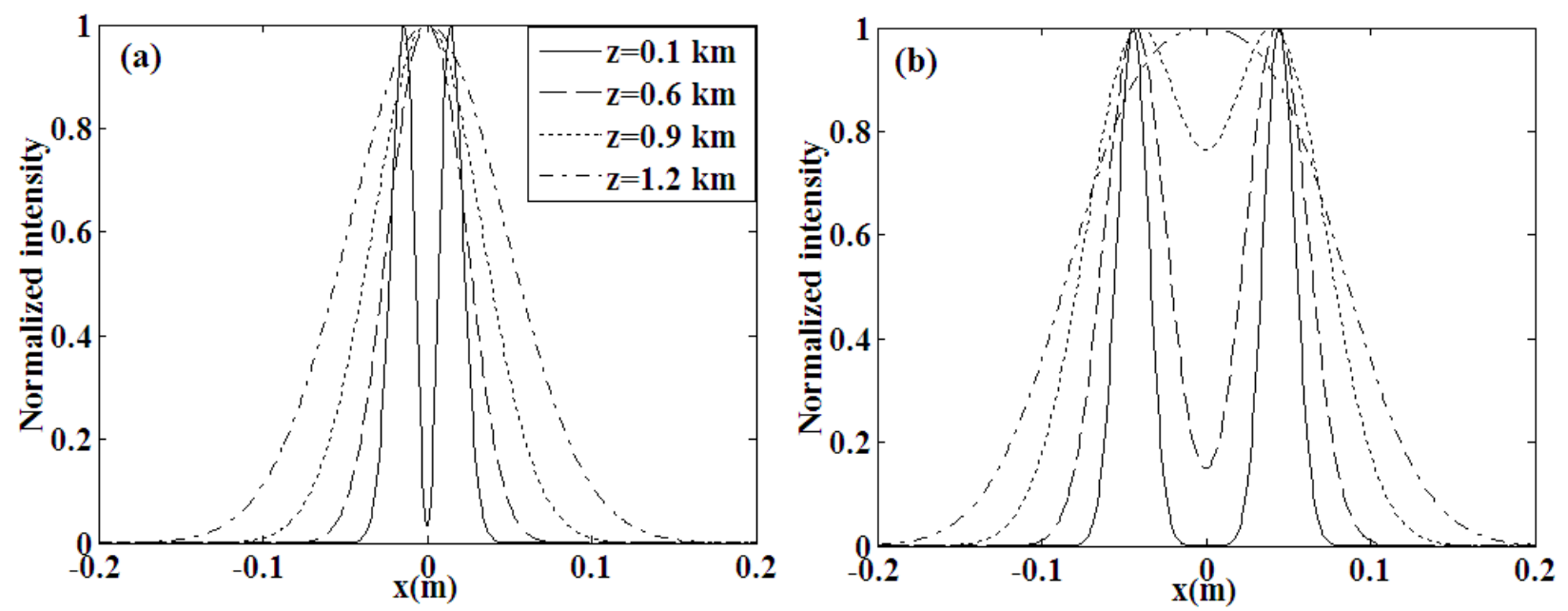

Figure 2: Normalized intensity in $\mathrm{x}$-direction of vChGB propagating through oceanic turbulence.

The parameters are the same as in Fig.1.

The effect of changing vortex charge $M$ on the spreading properties of a vChGB in the oceanic turbulence is illustrated in Figs. (3) and (4) for the small b and large b configurations, respectively. It can be seen that the rise speed of the central peak intensity becomes slower as $M$ is larger, this means that a beam with larger $M$ can keep its initial profile better than the one with smaller $M$. 

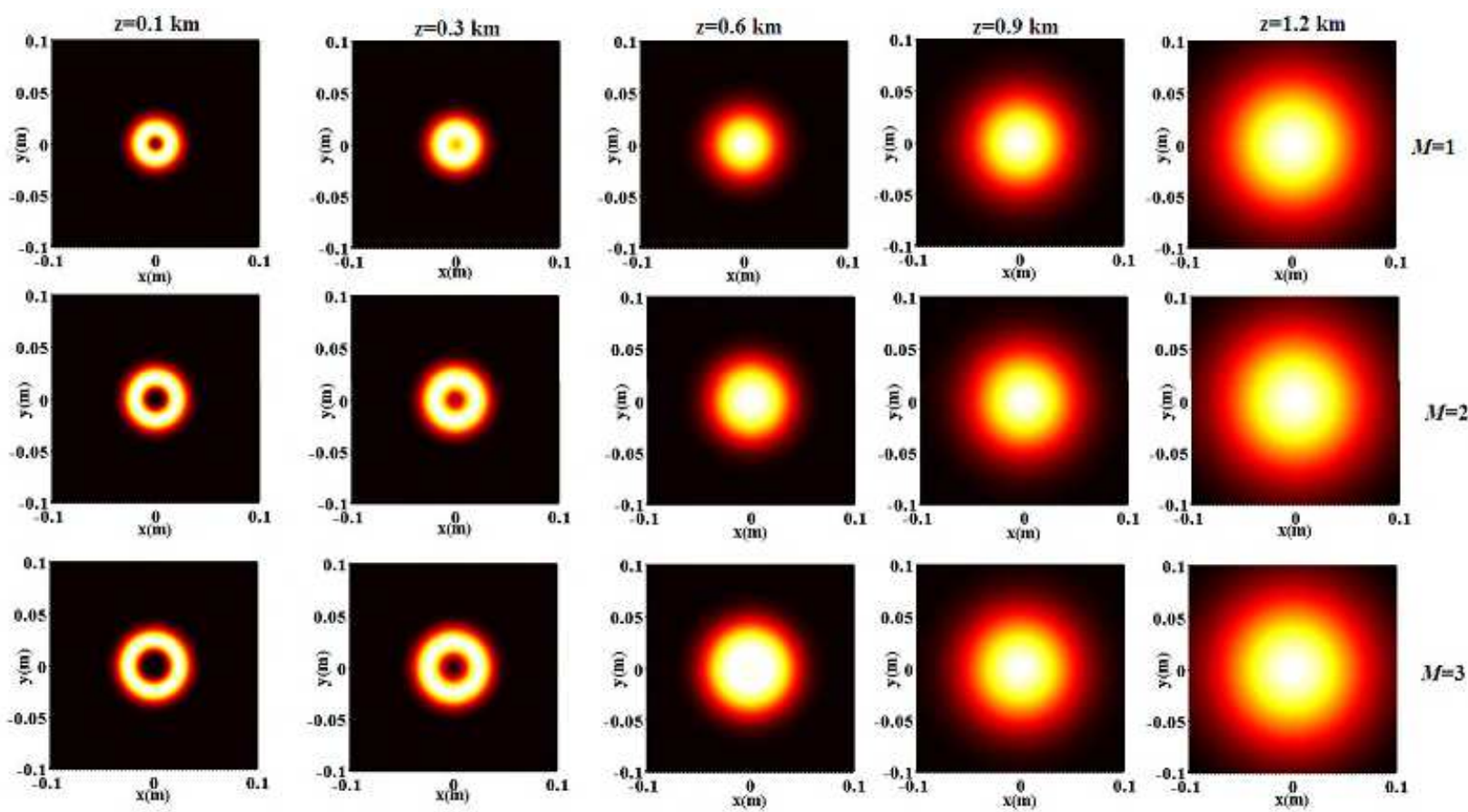

Figure 3: The average intensity of a vChGB in a oceanic turbulence for different values of the topological charge $M$ with $\omega_{0}=0.02 \mathrm{~m}, \lambda=417 \mathrm{~nm} \varepsilon=10^{-7}, \chi_{T}=10^{-9}, \varsigma=-2.5$ and $b=0.1$.
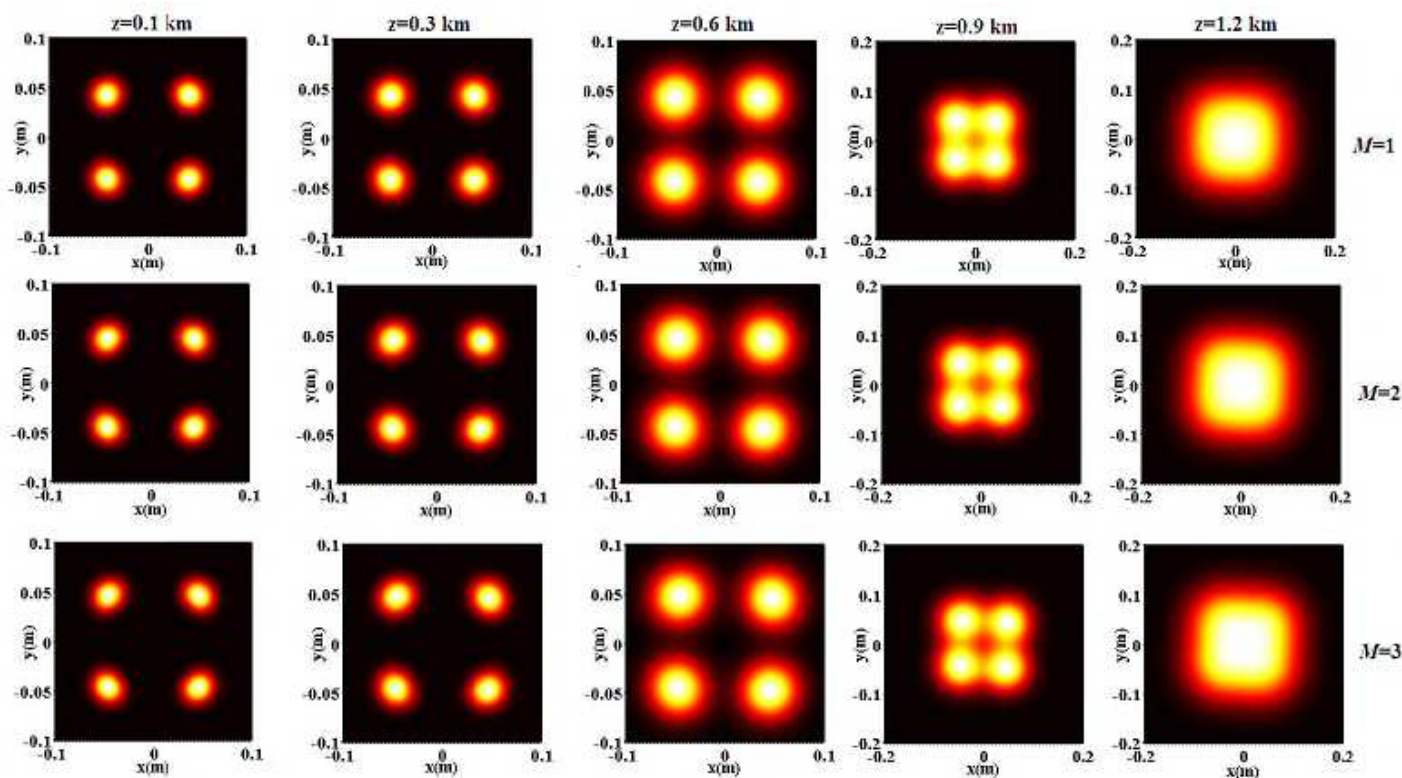

Figure 4: The same as Fig.3 except $b=4$.

For further analyzing the beam evolution properties in the turbulent medium, we have carried out in Fig. 5 the variation of the on-axis intensity as a function of propagation distance. The other parameters calculations are the same as in Figs. 3 and 4. It can be readily seen from the plots that 
the on-axis intensity vanishes at short propagation distances, and gradually increases with increasing the propagation process until it reaches a maximum value. Then, the central peak intensity decreases naturally with the propagation distance due to the diffraction process. It is clearly seen that for the beam with small b configuration (see Fig. 5(b)), the rise speed of the central peak intensity is slower when the topological charge $M$ is larger. Nevertheless, the on-axis intensity of the propagated beam is less sensitive to the value of $M$ for the large $b$ configuration.
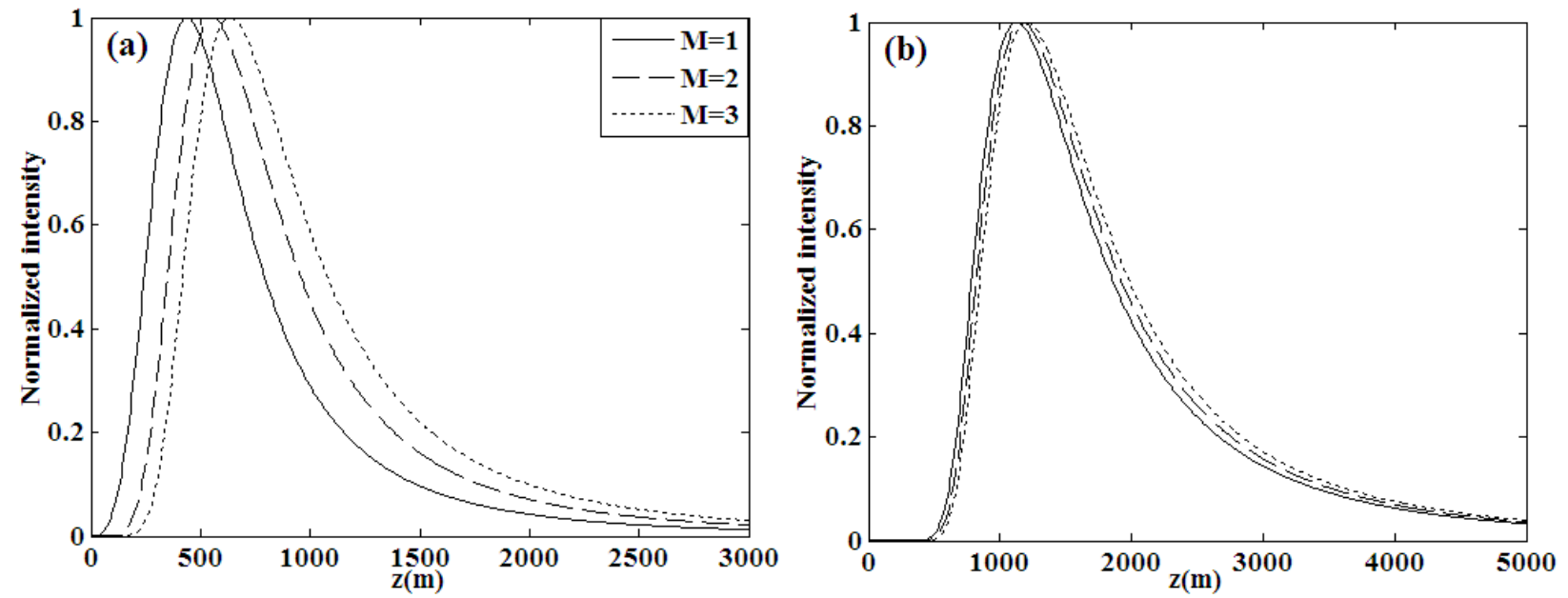

Figure 5: The normalized axial intensity versus propagation distance $\mathrm{z}$ of a vChGB in oceanic turbulence for different values of the b-parameter (a): $b=0.1$ and (b): $b=4$ versus the topological charge $M$ with $\omega_{0}=0.02 \mathrm{~m}, \lambda=417 \mathrm{~nm} \varepsilon=10^{-7}, \chi_{T}=10^{-9}$ and $\varsigma=-2.5$.

In order to investigate the influence of the strength of the oceanic turbulence on the propagation behaviour of a vChGB in the turbulent medium, we have depicted in Figs. (6), (7) and (8) the normalized on-axis intensity of the beam (with $M=1,2$ and 3) for different values of the sea water parameters $\left(\chi_{T}, \varsigma\right.$ and $\varepsilon$ ). From Figs. (6) and (7), it is shown that when the dissipation rate of mean-square temperature $\chi_{T}$ or the ratio of temperature to salinity $\varsigma$ increases, the rising speed of the central peak becomes faster until reaching the maximum value, and with further propagation distance the decline rate of the intensity curve is faster. In addition, it is found that the beam propagating in the turbulent ocean with larger value of the dissipation rate of turbulent kinetic energy per unit mass of fluid $\varepsilon$ evolves slower into Gaussian-like beam. 

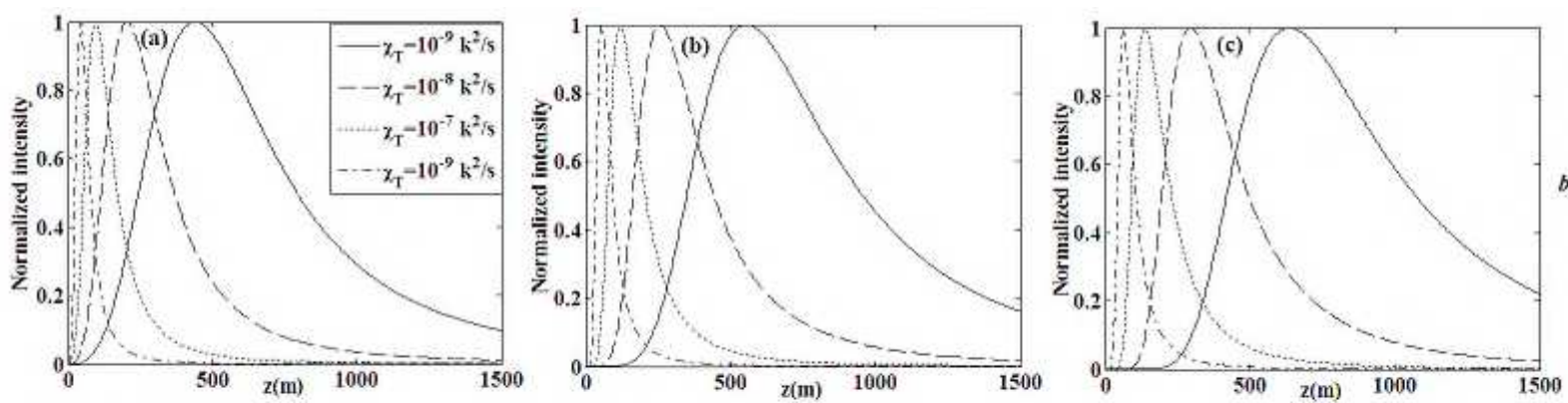

$b=0.1$
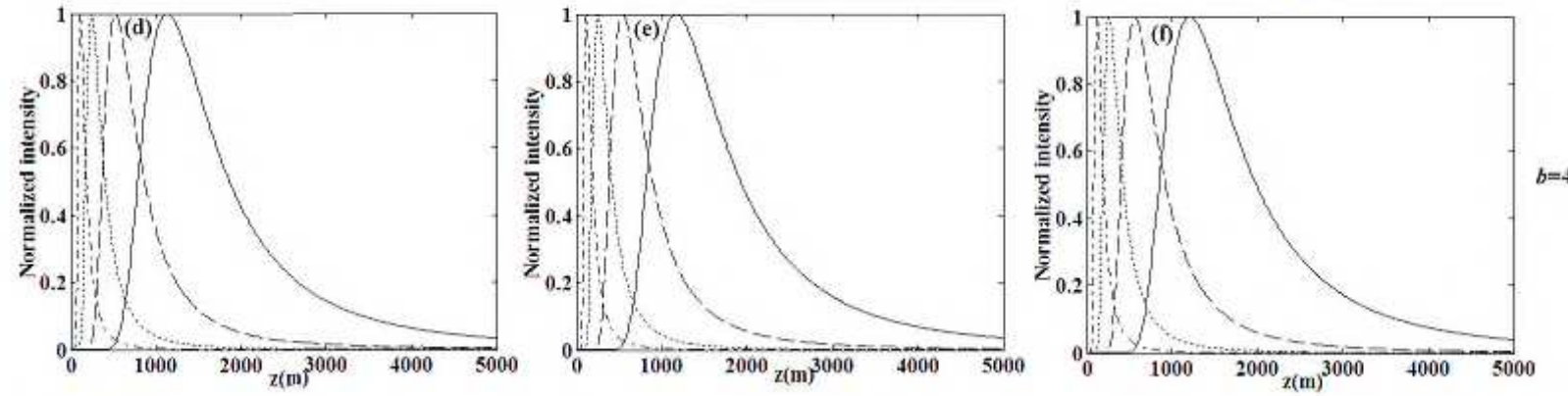

Figure 6: Normalized on-axis average intensity of a vChGB in oceanic turbulence versus $\mathrm{z}$ for different values of the rate of dissipation of mean-square temperature $\chi_{T}$, with $\omega_{0}=0.02 \mathrm{~m}, \lambda=417 \mathrm{~nm}, \varepsilon=10^{-7}$ and $\varsigma=-2.5$, rows (a-d) for $M=1$, (b-e) for $M=2$ and (c-f) for $M=3$.
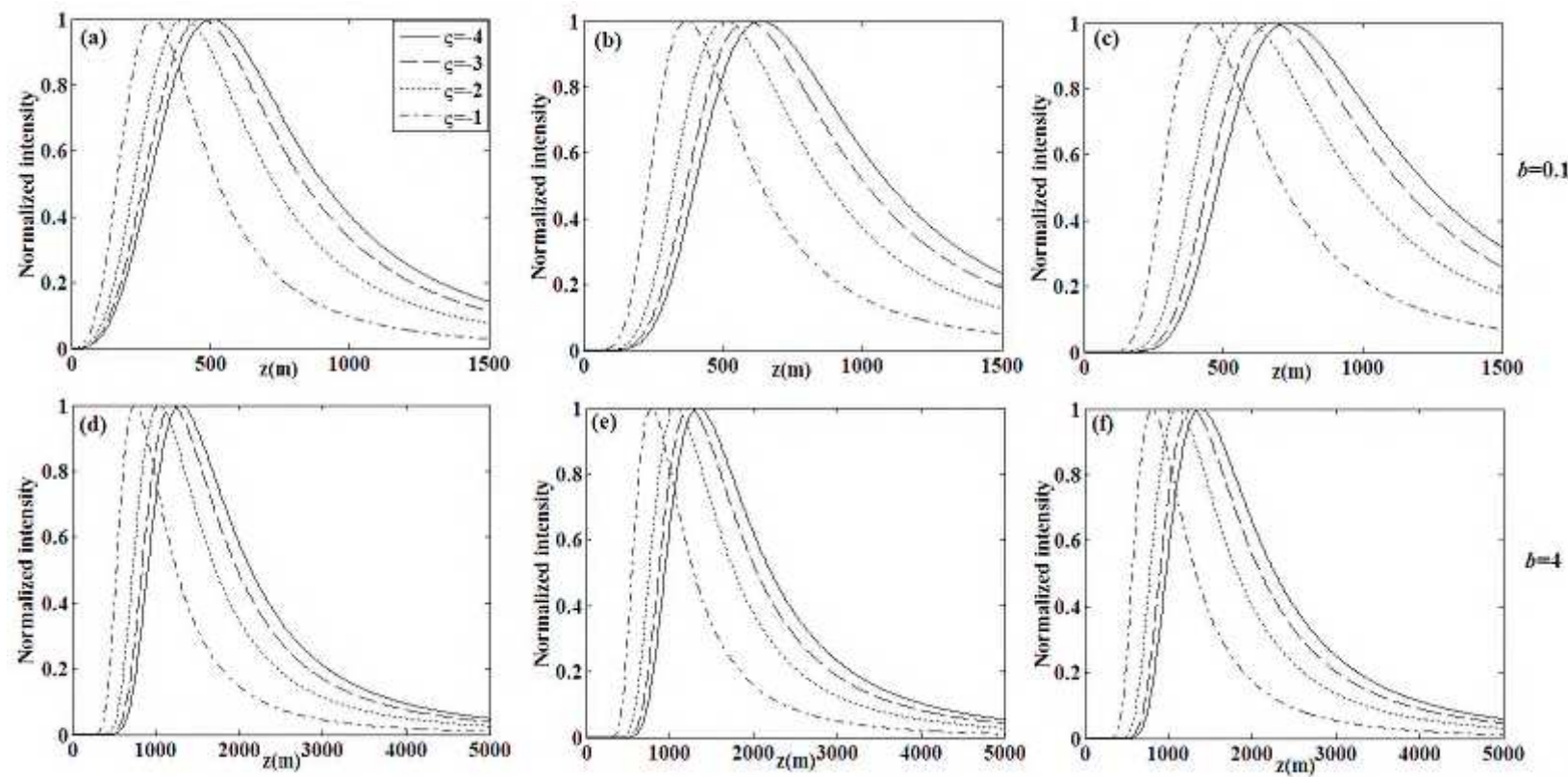

Figure 7: Normalized on-axis average intensity of the perturbed vChGB versus propagation distance $\mathrm{z}$ for different values of the ratio of temperature to salinity $\varsigma$, with $\omega_{0}=0.02 \mathrm{~m}, \lambda=417 \mathrm{~nm} \varepsilon=10^{-7}$, and $\chi_{T}=10^{-9}$, rows (a-d) for $M=1$, (b-e) for $M=2$ and (c-f) for $M=3$. 

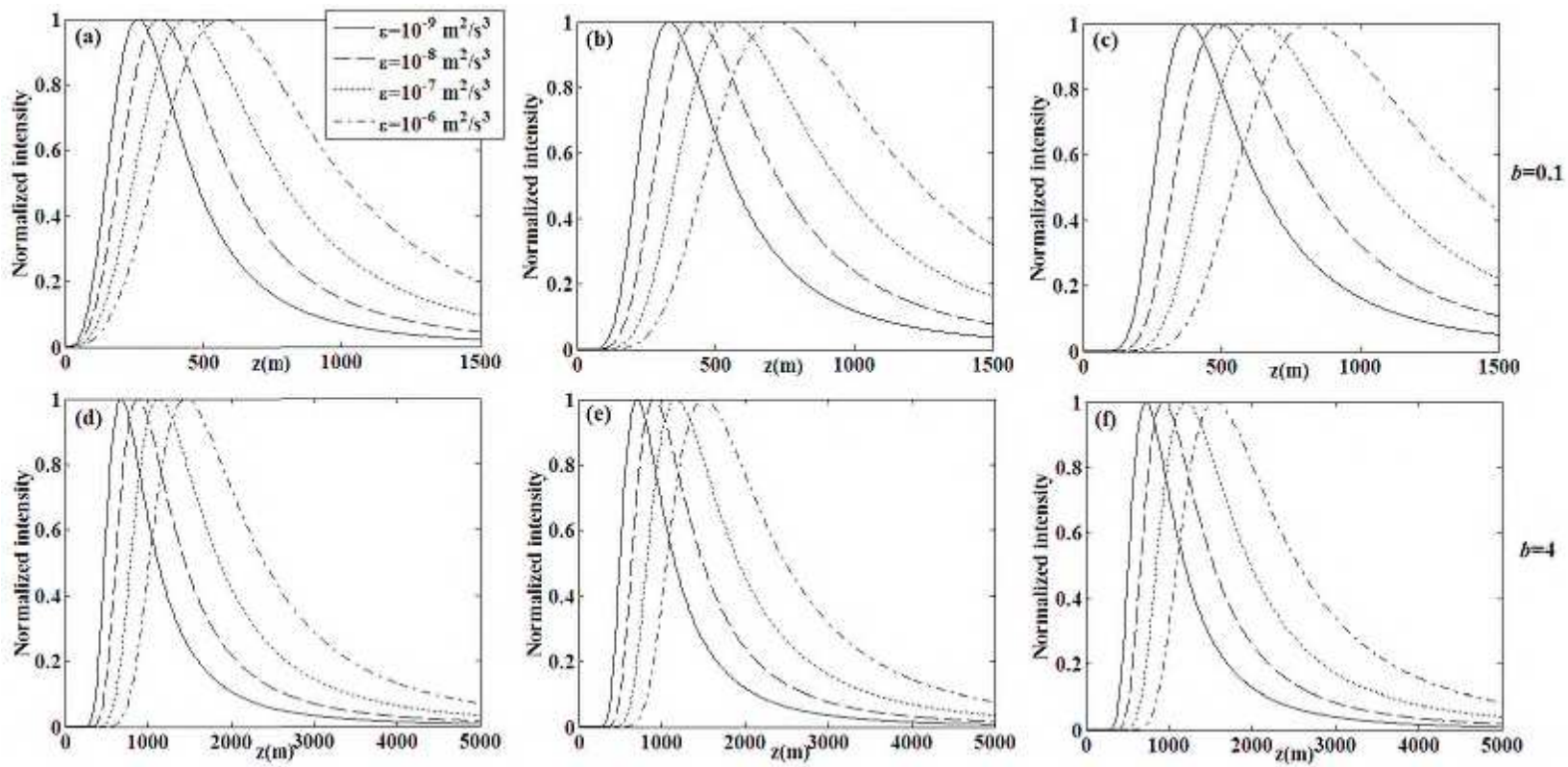

Figure 8: On-axis average intensity of the perturbed vChGB versus propagation distance z for different values of $\varepsilon$, rows (a-d) for $M=1$, (b-e) for $M=2$ and (c-f) for $M=3$.

The evolution of the normalized on-axis intensity of a vChGB propagating through oceanic turbulence versus the beam waist radius $\omega_{0}$ is depicted in Fig. 9.
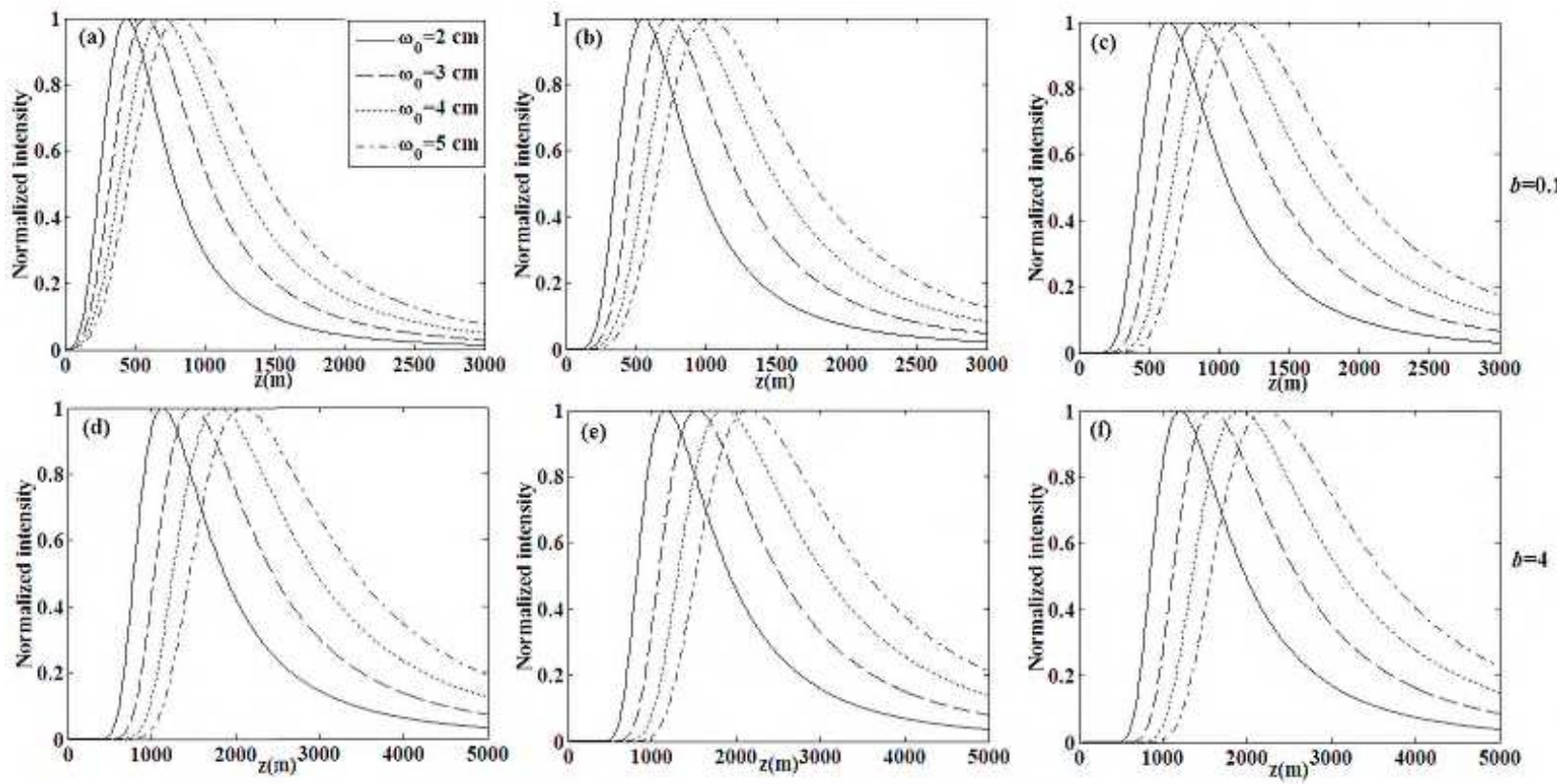

Figure 9: Normalized on-axis average intensity of a vChGB in oceanic turbulence versus propagation distance for different values of $\omega_{0}$, rows (a-d) for $M=1$, (b-e) for $M=2$ and (c-f) for $M=3$. 
It can be seen that the profile of evolution and the position of maximum of the on-axis intensity are shifted toward large propagation distance when $\omega_{0}$ is increased. In addition, one can note also that the intensity curve becomes wider as when $\omega_{0}$ is larger. This means that the beam can retain its dark centre longer, and the rise speed of the central peak intensity is slower when $\omega_{0}$ is larger. Thus increasing the beam waist may be beneficial for improving the transmission of the beam in oceanic turbulence.

Finally, the influence of wavelength $\lambda$ on the average intensity of a vChGB in oceanic turbulence is illustrated in Figs. 10 and 11, where we have depicted the evolutions of the normalized on-axis intensity versus the propagation distance for some values of $\lambda$ in the visible spectrum $(\lambda=417 \mathrm{~nm}$ , $488 \mathrm{~nm}, 532 \mathrm{~nm}$ and $633 \mathrm{~nm}$ ). From Fig. 10, one can note at first glance that the on-axis intensity is less sensitive to wavelength.
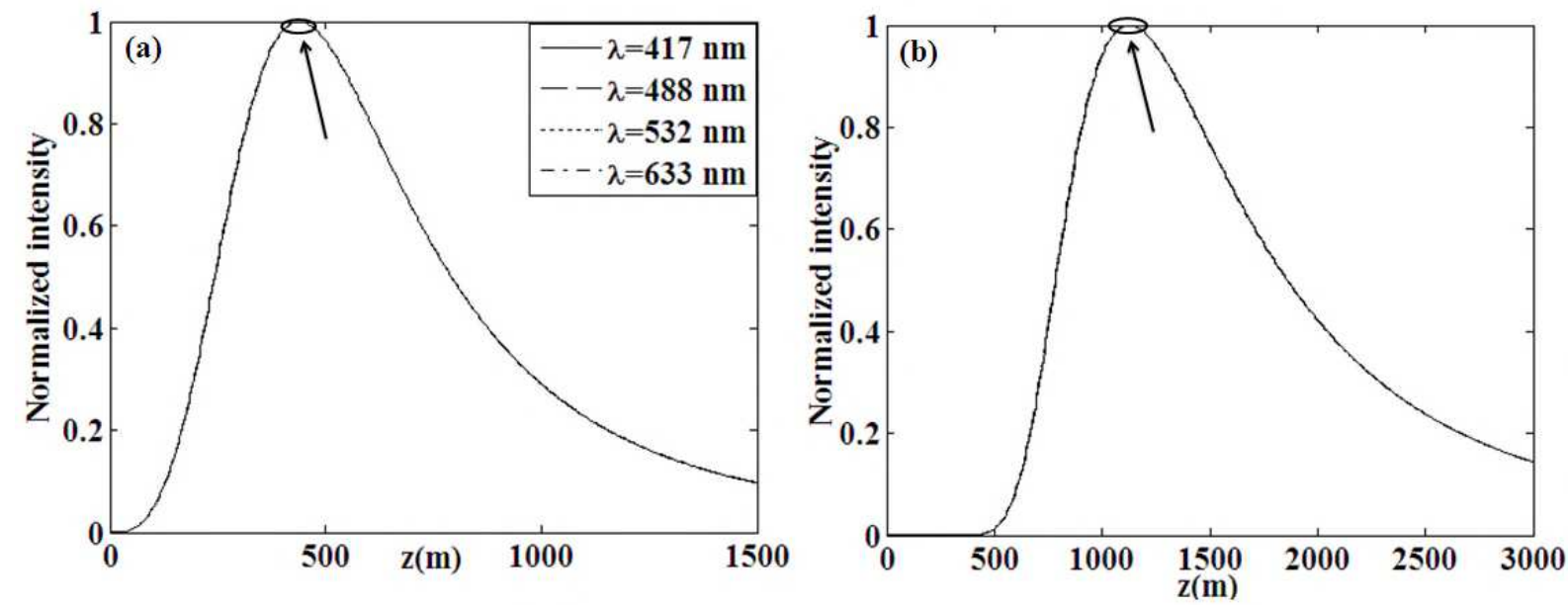

Figure 10: Normalized on-axis average intensity of a $\mathrm{vChGB}$ with $M=1$ in oceanic turbulence versus $\mathrm{z}$ for different values of $\lambda:$ (a) for $b=0.1$, (b) for $b=4$.

However, the zoom of the variation region shown in Fig. 11 indicates that the rise speed of the central peak intensity becomes slower and the intensity profile moves toward small propagation distance when the light wavelength is augmented. 

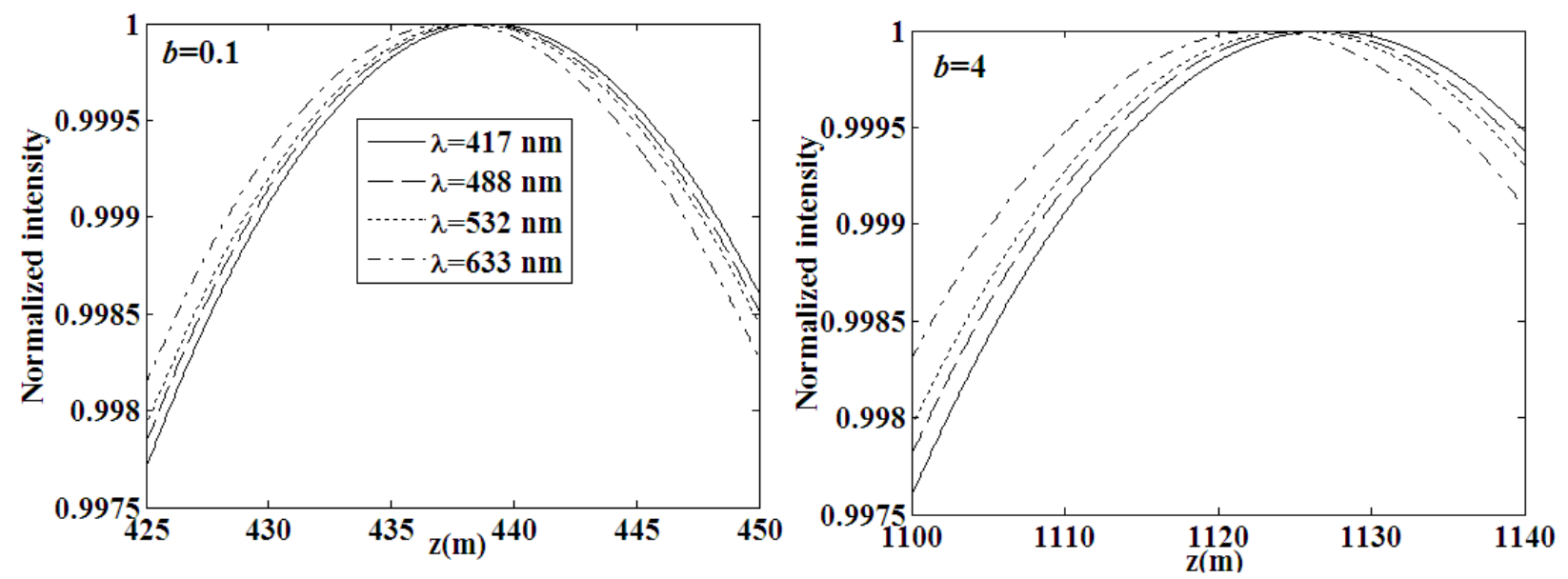

Figure 11: As in Fig.10 with zoom in on the region near maximum intensity position.

\section{Conclusion}

In summary, based on the extended Huygens-Fresnel diffraction integral and Rytov theory the propagation properties of a vChGB in oceanic turbulence are investigated theoretically and numerically. The analytical formula of the average intensity for the beam propagating in oceanic turbulence is derived in detail. The evolution behavior of the average intensity distribution for the beam propagating in oceanic turbulence is analyzed numerically as functions the turbulence strength, initial beam parameters, and propagation distance. The results reveal that a vChGB propagating in oceanic turbulence can keep its initial dark-hollow profile at short propagation distances, and evolves into Gaussian-like beam in far-field. It is shown that when the oceanic turbulence strength increases (i.e., by increasing the parameters $\chi_{T}$ and $\varsigma$ or decreasing the parameter $\varepsilon$ ) or the initial source parameters $b, \mathrm{M}, \omega_{0}$ and $\lambda$ decrease, the rising speed of the central peak becomes stronger. The results obtained in this paper would be conducive for further understanding the propagation properties of a vChGB in turbulent ocean, which are useful for optical underwater communication and remote sensing domain, imaging, and so on. 


\section{References}

[1] V.V. Nikishov, V.I. Nikishov, "Spectrum of turbulent fluctuations of the sea-water refraction index”. Int. J. Fluid Mech. Res. 27, 82-98 (2000).

[2] Y. Lacroix, D. Leandri, V.I. Nikishov, "Wave propagation in turbulent sea water". Int. J. Fluid Mech. Res, 38 (4), 366-386 (2010).

[3] M. Tang, D. Zhao, "Regions of spreading of Gaussian array beams propagating through oceanic turbulence”. Appl. Opt. 54, 3407-3411 (2015).

[4] Y. Baykal, "Scintillation of LED sources in oceanic turbulence”. Appl. Opt. 55 (31), 8860-8663 (2016).

[5] Y. Baykal, "Scintillation index in strong oceanic turbulence". Opt. Commun. 375, 15-18 (2016).

[6] M.M. Tang, D.M. Zhao, "Propagation of radially polarized beams in the oceanic turbulence". Appl. Phys. B 111, 665-670 (2013).

[7] Y.P. Huang, B. Zhang, Z.H. Gao, G.P. Zhao, Z.C. Duan, "Evolution behavior of Gaussian Schell-model vortex beams propagating through oceanic turbulence". Opt. Express 22, 1772317734 (2014).

[8] J. Xu, D.M. Zhao, "Propagation of a stochastic electromagnetic vortex beam in the oceanic turbulence”. Opt. Laser Technol. 57, 189-193 (2014).

[9] D.J. Liu, Y.C. Wang, H.M. Yin, "Evolution properties of partially coherent flat-topped vortex hollow beam in oceanic turbulence”. Appl. Opt. 54, 10510-10516 (2015).

[10] T.Yang, X.L. Ji, X.Q. Li, "Propagation characteristics of partially coherent decentred annular beams propagating through oceanic turbulence”. Acta Phys. Sin. 64, 204206 (2015).

[11] Y.P. Huang, P. Huang, F.H. Wang, G.P. Zhao, A.P. Zeng, "The influence of oceanic turbulence on the beam quality parameters of partially coherent Hermite-Gaussian linear array beams". Opt. Commun. 336, 146-152 (2015).

[12] Y. Li, Y. Han, Z. Cui, "On-axis average intensity of a hollow Gaussian beam in turbulent ocean”. Opt. Eng. 58 (9), 096115-096121 (2019).

[13] C. Ding, L. Liao, H. Wang, Y. Zhang, L. Pan, “Effect of oceanic turbulence on the propagation of cosine-Gaussian-correlated Schell-model beams". J. Opt. 17, 035615-035623 (2015).

[14] D. Liu, H. Yin, G. Wang, Y. Wang, "Propagation of partially coherent Lorentz-Gauss vortex beam through oceanic turbulence”. Appl. Opt. 56, 8785-8792 (2017).

[15] D. Liu, Y. Wang, G. Wang, X. Luo, H. Yin, “ Propagation properties of partially coherent 
four-petal Gaussian vortex beams in oceanic turbulence”. Laser Phys. 27, 016001-016008 (2017). [16] D. Liu, Y. Wang, "Properties of a random electromagnetic multi-Gaussian Schell-model vortex beam in oceanic turbulence”. Appl. Phys. B 124, 176-184 (2018).

[17] Y. Baykal, "Intensity correlation of flat-topped beams in oceanic turbulence”. J. Mod. Optics 67(9), 799-804 (2020).

[18] J. Wang, X. Wang, Q. Peng, S. Zhao, "Propagation characteristics of autofocusing Airy beam with power exponential phase vortex in weak anisotropic oceanic turbulence”. J. of Mod. Optics 68 (19), 1059-1065 (2021).

[19] Z. Hricha, M. Yaalou, A. Belafhal, "Introduction of a new vortex cosine-hyperbolic-Gaussian beam and the study of its propagation properties in Fractional Fourier Transform optical system". Opt. Quant. Elec. 52, 296-302 (2020).

[20] G. Zhou, Y. Cai, C. Dai, “Hollow vortex Gaussian beams”. Sci. Chin., 56 (5), 896-903 (2013). [21] L.W. Casperson, D.G. Hall, and A. A. Tovar, "Sinusoidal-Gaussian beams in complex optical systems," J. Opt. Soc. Am. A 14, 3341-3348 (1997).

[22] A.E. Siegman, "Lasers”. University Science Books, (1986).

[23] Z. Hricha, M. Lazrek, M. Yaalou, A. Belafhal, "Propagation of vortex cosine-hyperbolicGaussian beams in atmospheric turbulence”. Opt. Quant. Elec. 53 (8), 383-398 (2021).

[24] M. Lazrek, Z. Hricha, A. Belafhal, "Partially coherent vortex cosh-Gaussian beam and its paraxial propagation”. Accepted in Opt. Quant. Elec. (October, 2021).

[25] M. Born, E. Wolf, "Principles of Optics". Seventh (expanded) ed, Cambridge University Press, Cambridge, UK (1999).

[26] L.C. Andrews, R.L. Philips, "Laser beam propagation through Random media". SPIE Press, Washington (1998).

[27] M. Abramowitz, I.A. Stegun (Eds.), "Handbook of Mathematical Functions”. Nat. Bureau of Standards Washington, DC (1964).

[28] A. Belafhal, Z. Hricha, L. Dalil-Essakali, T. Usman, "A note on some integrals involving Hermite polynomials and their applications”. Adv. Math. Mod. and App. 5 (3), 313-319 (2020). [29] I.S. Gradshteyn, I.M. Ryzhik, "Tables of Integrals, Series, and Product”. Fifth ed., Academic Press, New York (1994). 